\title{
Measured LTE Throughput for SISO, SIMO and MIMO in Polarization-Random-LOS
}

\author{
Madeleine Schilliger Kildal*, Jan Carlsson*†, Andrés Alayón Glazunov*, Per-Simon Kildal* \\ ${ }^{*}$ Department of Signals and Systems, Chalmers University of Technology, Gothenburg, Sweden \\ ${ }^{\dagger}$ Electronics department, SP Technical Research Institute of Sweden, Borås, Sweden
}

\begin{abstract}
LTE throughput measurements in a Random Lineof-Sight (Random-LOS) setup have been performed. The effect of polarization-randomness on the level of detection threshold of one or two bitstreams has been investigated. Good agreement is shown between theory and experiments for the SISO, SIMO and MIMO cases.
\end{abstract}

\section{INTRODUCTION}

The Rich Isotropic Multipath (RIMP) environment has become a well known environment for characterizing small active wireless devices, such as mobile phones [1]. When moving towards higher frequencies the Line-Of-Sight (LOS) propagation conditions will dominate. In these LOS environments the receiver antenna (or rather the user terminal) will introduce randomness into the propagation channel due to unpredictable movements and orientation [2]. This we call a Random-LOS environment where both the Angle of Arrival (AoA) and the polarization can be random. In this paper we investigate the effect of the polarization-randomness and keep the AoA fixed.

\section{METHOD}

The measurements of throughput in Polarization-RandomLOS have been performed in an anechoic chamber (AC) (dimensions $2.0 \mathrm{~m} \times 3.5 \mathrm{~m} \times 5.5 \mathrm{~m}$ ). As an antenna under test (AUT) we use a differentially excited 2-port bowtie antenna [3], see Fig. 1a. A quadridge horn antenna (ETS Lindgren Open Boundary Quadridge Horn, Model 3164-05) was used as the chamber antenna, see Fig. 1b. The antennas were placed at the same height and at a distance of $3.8 \mathrm{~m}$ from each other.

The bowtie antenna was conductively connected to a twoport LTE USB modem placed inside a shielded box, to avoid that the internal antennas in the modem were receiving any signal. The LTE throughput measurements were performed with a Rohde \& Schwarz CMW500 communication tester on LTE band 7, channel 3100, with a dowlink frequency of $2.655 \mathrm{GHz}$. The measurements were performed with a downlink modulation of 16-QAM.

The maximum theoretical and measured throughput was $5.738 \mathrm{Mbps}$ for one bitstream, i.e., Single Input Single Output (SISO) and Single Input Multiple Output (SIMO) cases. For two bitstreams, i.e., Multiple Input Multiple Output (MIMO) case, the maximum throughput was $11.476 \mathrm{Mbps}$. Reference measurements with the bowtie setup were performed with a vector network analyzer (VNA) to calibrate out the path loss and other set up losses.

The Polarization-Random-LOS measurements were performed by facing the bowtie antenna towards the chamber antenna. The polarization of the chamber antenna was changed by rotating it around its $z$-axis from $-90^{\circ}$ to $+90^{\circ}$, where $0^{\circ}$ corresponds to aligning port 1 on the bowtie with the transmitting port on the chamber antenna. One whole throughput curve is measured at every $10^{\circ}$ angle, by sweeping the power such that we get a throughput ranging from $100 \%$ to $0 \%$ throughput. This throughput curve will abruptly go from $100 \%$ to $0 \%$ in an interference-free measurement environment. However, the used AC was not well shielded. Hence, to see the trends more clearly, each measurement is repeated three times. For the SISO measurements a $3 \mathrm{~dB}$ attenuator is added at the receive side to further reduce the interference coming in through the antenna.

\section{Measurement Results}

Fig. 1c-1i illustrate the threshold power estimated at the $50 \%$ level of the detection threshold [4] as a function of polarization angle. This is called the $50 \%$ level of detection threshold. In Fig. 1c there is also a theoretical $\cos ^{2} \phi$ curve representing the polarization mismatch fitted to the measured results.

If we assume that in the receive diversity case (SIMO), maximal ratio combining (MRC) is used, we would expect that any polarization can be detected equally well as the best SISO case. Under the condition that the signals (waves) received on the two ports are perfectly orthogonal and with the same amplitude [5]. The degradation of the system can clearly be seen when we have amplitude imbalance. We achieve the amplitude imbalance by a $3 \mathrm{~dB}$ attenuator on one of the receiving ports. A theoretical MRC curve with an amplitude imbalance of $3 \mathrm{~dB}$ is fitted to the measured SIMO results in Fig. $1 \mathrm{e}$ and $1 \mathrm{f}$.

The MIMO curve in Fig. $1 \mathrm{~g}$ shows a $3.8 \mathrm{~dB} \pm 0.8 \mathrm{~dB}$ degradation as compared to the SIMO measurement, since the available power is divided on two bitstreams and theoretically gives a degradation of $3 \mathrm{~dB}$ [5]. When adding an amplitude imbalance of $3 \mathrm{~dB}$ between the two MIMO ports we would assume to get an average of $75 \%$ worse performance, which corresponds to $-1.25 \mathrm{~dB}$. For the measurements, see Fig $1 \mathrm{~h}$ and Fig 1i. we get an average performance degradation of $-0.7 \mathrm{~dB} \pm 0.8 \mathrm{~dB}$. 


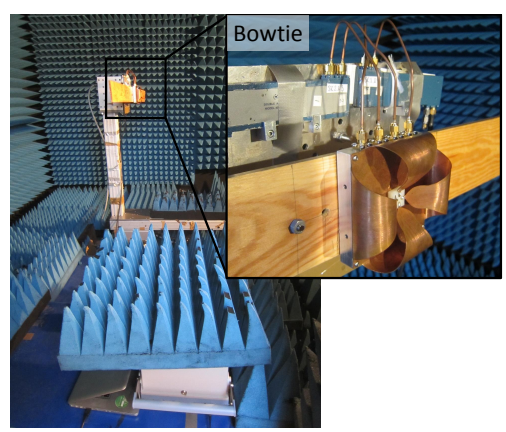

(a)

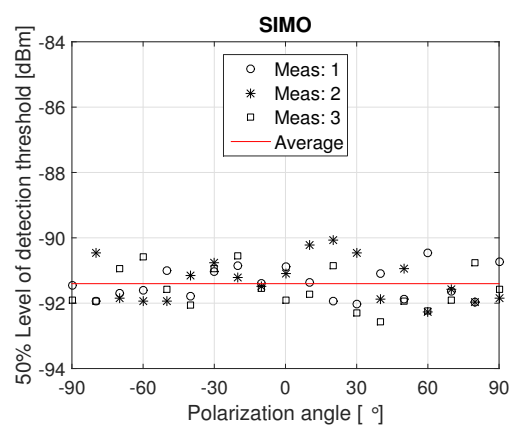

(d)

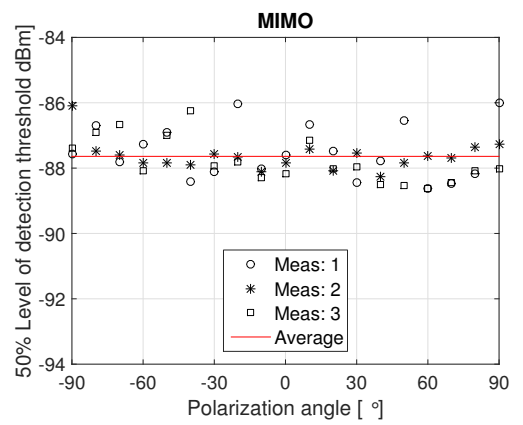

(g)

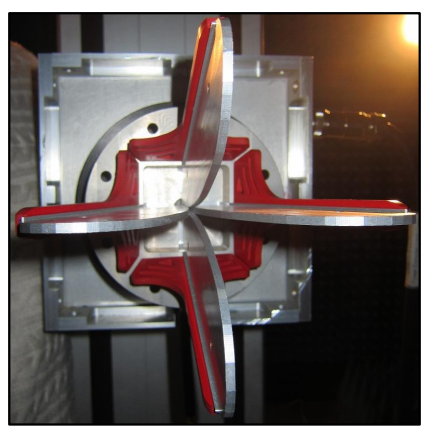

(b)

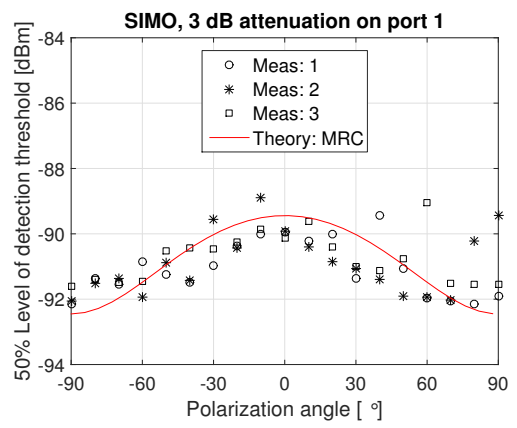

(e)

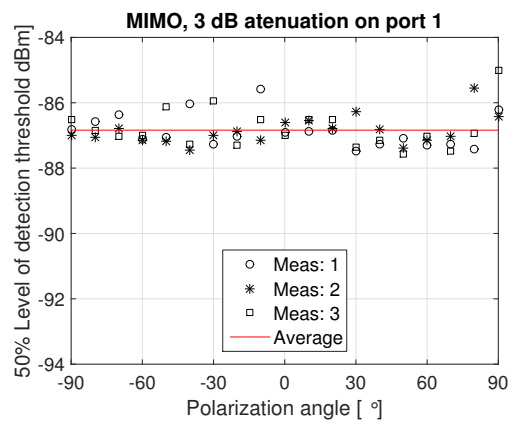

(h)

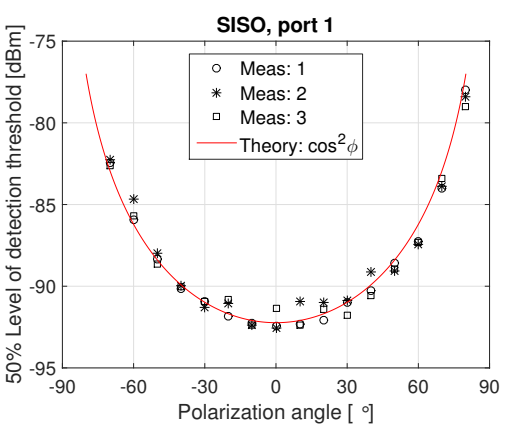

(c)

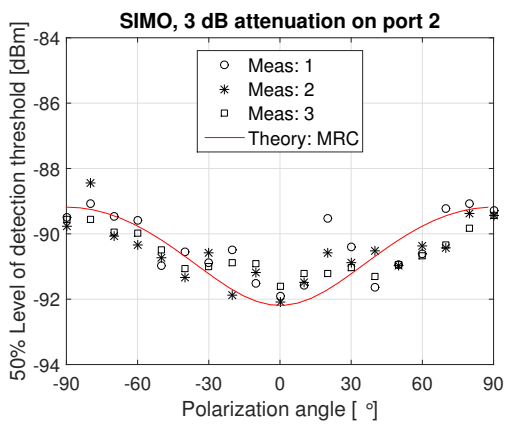

(f)

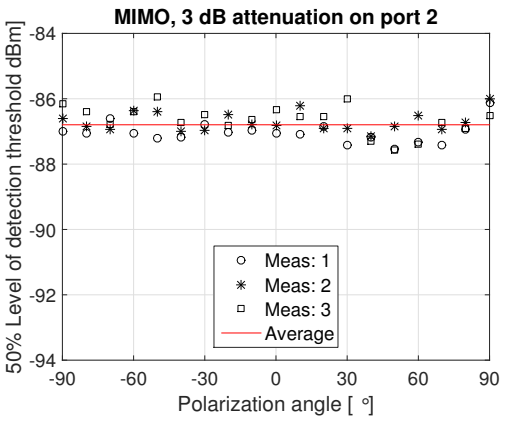

(i)

Fig. 1. Polarization-Random-LOS measurements. (a) shows the measurement setup in the AC together with the bowtie antenna, (b) shows the quadridge horn antenna and (c) - (i) shows the SISO, SIMO and MIMO measurement results.

\section{CONCLusions}

We see that we can evaluate the Polarization-Random-LOS well in this setup, where the measured Polarization-RandomLOS results fit well the expected results. We see that the SISO case has an expected $\cos ^{2} \phi$-dependency due to polarization mismatch. The SIMO case with orthogonal receive antennas can detect any linear polarization equally well and the MIMO case has a $3.8 \mathrm{~dB} \pm 0.8 \mathrm{~dB}$ higher detection threshold than SIMO due to the division of the power between two bitstreams. For further improving the results and reduce the interference, one could measure with attenuators on the receiving ports.

\section{ACKNOWLEDGEMENT}

The project is partly supported by the Swedish Research Council VR, through an industrial $\mathrm{PhD}$ project.

\section{REFERENCES}

[1] P.-S. Kildal, C. Orlenius, and J. Carlsson, "OTA Testing in Multipath of Antennas and Wireless Devices With MIMO and OFDM," Proceedings of the IEEE, vol. 100, no. 7, pp. 2145-2157, Jul. 2012.

[2] P.-S. Kildal, "Preparing for GBit/s Coverage in 5g: Massive MIMO, PMC Packaging by Gap Waveguides, OTA Testing in Random-LOS," in 2015 Loughborough Antennas \& Propagation Conference, Nov. 2015.

[3] H. Raza, A. Hussain, J. Yang, and P.-S. Kildal, "Wideband Compact 4Port Dual Polarized Self-Grounded Bowtie Antenna," IEEE Transactions on Antennas and Propagation, vol. 62, no. 9, pp. 4468-4473, Sep. 2014.

[4] M. S. Kildal, J. Kvarnstrand, J. Carlsson, A. A. Glazunov, A. Majidzadeh, and P.-S. Kildal, "Initial Measured OTA Throughput of 4g LTE Communication to Cars with Roof-Mounted Antennas in 2d Random-LOS," in 2015 International Symposium on Antennas and Propagation (ISAP), Nov. 2015.

[5] A. Razavi, A. A. Glazunov, P.-S. Kildal, and J. Yang, "Characterizing Polarization-MIMO Antennas in Random-LOS Propagation Channels," unpublished. 